\title{
New Travelling-Wave Solutions for Dodd-Bullough Equation
}

\author{
Guicheng Shen, ${ }^{1}$ Yunchuan Sun, ${ }^{2}$ and Yongping Xiong ${ }^{3}$ \\ ${ }^{1}$ School of Information, Beijing Wuzi University, Beijing 101149, China \\ ${ }^{2}$ Beijing Normal University, Beijing 100875, China \\ ${ }^{3}$ State Key Laboratory of Networking and Switching Technology, Beijing University of Posts and Telecommunications, \\ Beijing 100876, China
}

Correspondence should be addressed to Yunchuan Sun; yunch@bnu.edu.cn

Received 2 April 2013; Accepted 23 May 2013

Academic Editor: Shiping Lu

Copyright (C) 2013 Guicheng Shen et al. This is an open access article distributed under the Creative Commons Attribution License, which permits unrestricted use, distribution, and reproduction in any medium, provided the original work is properly cited.

A new method, which assumes $\phi^{\prime}$ with a function form of $f\left(e^{\phi}\right)$, is applied to solve the Dodd-Bullough equation $\phi_{u v}=e^{\phi}-e^{-2 \phi}$ through travelling-wave transformation. A new family of explicit travelling wave solutions is derived. The proposed method works efficiently to be applied to solve other forms of Dodd-Bullough equations.

\section{Introduction}

The Dodd-Bullough equation

$$
\phi_{u v}=e^{\phi}-e^{-2 \phi},
$$

initiated by Dodd and Bullough [1] and Žiber and Šabat [2], plays a significant role in many scientific applications such as solid state physics, nonlinear optics, and quantum field theory.

There are many research works for Dodd-Bullough equation in the last decades. It is shown that Dodd-Bullough determines the intrinsic geometry of the two-dimensional affine sphere in the three-dimensional unimodular affine space [3]. It has also been shown that the Dodd-Bullough equation is related to the nonlinear two-dimensional $\mathrm{SL}(3, \mathrm{R})$ sigma model in [4]. To solve the Dodd-Bullough equation, many mathematicians have put forward some methods, and different forms of solution formulas have been retrieved. Reference [5] develops two-soliton solution formula and $\mathrm{N}$-soliton solution formula by means of dressing the trivial one $\phi \equiv 0$. The dressing procedure for (1) is also suggested in [6]. By using three Weierstrass functions, [7] proposes some explicit solution formulas. Reference $[8,9]$ obtain some general ineffective formulas for solutions of (1), but no concrete solutions. The MSE (modified simple equation) method has been proposed in [10-12] to explore the exact traveling solution of nonlinear physical systems, then [13] applies the MSE method to the Tzitzeica-Dodd-Bullough equation for some new solutions.

Tanh method is efficient to deal with many kinds of nonlinear equations. Applying the tanh method to the DoddBullough-Mikhailov and the Tzitzeica-Dodd-Bullough equations, [14] derives some solitons and periodic solutions. By developing an extended tanh method, [15] builds some new explicit solutions for Dodd-Bullough equation.

This paper aims to propose a new method to deal with the Dodd-Bullough equation, which is based on an assumption that the first order differential of $\phi$ on the travelling wave variable $\xi=u+c v$. A new family of explicit solutions for (1) is derived. The proposed method works efficiently to solve other forms of Dodd-Bullough equations. We also compare some of our results to some previous solutions and find that the solution of the Dodd-Bullough-Mikhailov equation given in [14] is only a special case of ours. Moreover, our solutions for Tzitzeica-Dodd-Bullough equations are different from previous results in [13] and other works.

\section{The Proposed Method}

Our method aims to solve some nonlinear equations with the form (2), after the travelling-wave transformation,

$$
F\left(\phi^{\prime \prime}\right)=G(g(\phi)),
$$


where $F, G$, and $g$ might be any functions. The technique is based on a priori assumption: the traveling-wave solutions of (2) satisfy $\phi^{\prime}$ which can be expressed in a form of some function of $g(\phi)$.

The main steps of our proposed method are as follows.

(1) We unite the independent variables $u$ and $v$ into one wave variable $\xi=u+c v$ to carry out the PDE in two variables,

$$
P\left(\phi_{u v}, \phi_{u v}^{\prime}, \phi_{u v}^{\prime \prime}, \ldots\right)=0,
$$

into an ODE,

$$
O\left(\phi(\xi), \phi^{\prime}(\xi), \phi^{\prime \prime}(\xi), \ldots\right)=0
$$

The precondition for our method is (4) which meets a form of (2) after some transformation.

(2) To find the travelling-wave solutions of (2), we take an assumption that

$$
\phi^{\prime}=f(g(\phi))
$$

then we get

$$
\phi^{\prime \prime}=f^{\prime}(g(\phi)) g^{\prime}(\phi) \phi^{\prime}
$$

According to the assumption, obviously,

$$
\phi^{\prime \prime}=f(g(\phi)) f^{\prime}(g(\phi)) g^{\prime}(\phi),
$$

then, substitute (7) into (2),

$$
F\left(f(g(\phi)) f^{\prime}(g(\phi)) g^{\prime}(\phi)\right)=G(g(\phi)) .
$$

Let $\psi=g(\phi)$, then we obtain that

$$
F\left(f(\psi) f^{\prime}(\psi) \psi^{\prime}\right)=G(\psi)
$$

(3) Try to retrieve the form of the function $f$ by solving (9). In some cases, (9) is a variable separated ODE.

(4) Once the form of the function $f$ is retrieved, return to (5), and we can get the solutions of $\phi$ by integration because (5) is also a variable separated ODE of the solutions, actually;

$$
\begin{gathered}
\frac{d \phi}{f(g(\phi))}=d \xi \\
\int \frac{d \phi}{f(g(\phi))}=\int d \xi=\xi+P,
\end{gathered}
$$

where $P$ is an integration constant.

In some cases, the explicit solutions can be retrieved by seeking the inverse function. Otherwise, only the implicit can be derived if the integration of the left side of (11) is much complex.

\section{Solutions for Dodd-Bullough Equation}

For the Dodd-Bullough given by (1), after travelling-wave transformation via $\xi=u+c v$, we can get that

$$
c \phi^{\prime \prime}=e^{\phi}-e^{-2 \phi} .
$$

Next, we assume that $\phi^{\prime}(\xi)$ meets an ODE form of some functions of $e^{\phi}$ for $\phi^{\prime \prime}(\xi)$ which satisfies a simple function form of $e^{\phi}$ :

$$
\phi^{\prime}=f\left(e^{\phi}\right)
$$

Following (6), we can get that

$$
\phi^{\prime \prime}=f\left(e^{\phi}\right) f^{\prime}\left(e^{\phi}\right) e^{\phi} .
$$

Consequently, by substituting (14) into (12), we obtain that

$$
c f\left(e^{\phi}\right) f^{\prime}\left(e^{\phi}\right) e^{\phi}=e^{\phi}-e^{-2 \phi} .
$$

Considering $\phi^{\prime}$ is a function of $e^{\phi}$, let $e^{\phi}=\psi$, then $f$ is a function of $\psi$.

Now, we try to get the form of the function $f$ :

$$
\begin{gathered}
c f(\psi) f^{\prime}(\psi) \psi=\psi-\psi^{-2}, \\
c f(\psi) f^{\prime}(\psi)=1-\psi^{-3} .
\end{gathered}
$$

Equation (17) is an ODE of variable separated:

$$
\frac{c}{2} f^{2}(\psi)=\psi+\frac{1}{2} \psi^{-2}+P
$$

where $P$ is a constant of integration,

$$
f(\psi)= \pm \sqrt{\frac{2}{c}\left(\psi+\frac{1}{2} \psi^{-2}+P\right)} .
$$

Remembering that $e^{\phi}=\psi$, then $\phi=\ln \psi$,

$$
\phi^{\prime}=\frac{\psi^{\prime}}{\psi} \text {. }
$$

From (13), obviously,

$$
f(\psi)=\frac{\psi^{\prime}}{\psi} .
$$

By comparing (19) and (21), we get

$$
\frac{\psi^{\prime}}{\psi}= \pm \sqrt{\frac{2}{c}\left(\psi+\frac{1}{2} \psi^{-2}+P\right)}
$$

that is,

$$
\frac{d \psi}{d \xi}=\psi^{\prime}= \pm \psi \sqrt{\frac{2}{c}\left(\psi+\frac{1}{2} \psi^{-2}+P\right)} .
$$


Equation (23) is a variable separated ODE; using a symbol computation software program, such as MATHEMATICA, we obtain that

$$
\begin{aligned}
& \xi+Q \\
& = \pm\left(2 \text { Elliptic } F\left(\arcsin \left(\sqrt{\frac{-\psi+r_{3}}{-r_{2}+r_{3}}}\right), \frac{r_{2}-r_{3}}{r_{1}-r_{3}}\right)\right. \\
& \left.\times \sqrt{\psi-r_{3}} \sqrt{\frac{\psi-r_{1}}{-r_{1}+r_{3}}} \sqrt{\frac{\psi-r_{2}}{-r_{2}+r_{3}}}\right) \\
& \times\left(\psi \sqrt{\frac{1+2 P \psi^{2}+2 \psi^{3}}{c \psi^{2}}} \sqrt{\frac{\psi-r_{3}}{r_{2}-r_{3}}}\right)^{-1} \\
& = \pm\left(2 \sqrt{c\left(\psi-r_{3}\right)\left(r_{2}-r_{3}\right)}\right. \\
& \left.\times \sqrt{\frac{\left(\psi-r_{1}\right)\left(\psi-r_{2}\right)}{\left(-r_{1}+r_{3}\right)\left(-r_{2}+r_{3}\right)}}\right) \\
& \times\left(\sqrt{1+2 P \psi^{2}+2 \psi^{3}}\right)^{-1} \\
& \times \text { EllipticF }\left(\arcsin \sqrt{\frac{-\psi+r_{3}}{-r_{2}+r_{3}}}, \frac{r_{2}-r_{3}}{r_{1}-r_{3}}\right) \\
& = \pm\left(2 \sqrt{\frac{c\left(r_{2}-r_{3}\right)\left(\psi-r_{3}\right)\left(\psi-r_{1}\right)\left(\psi-r_{2}\right)}{\left(-r_{1}+r_{3}\right)\left(-r_{2}+r_{3}\right)}}\right) \\
& \times\left(\sqrt{1+2 P \psi^{2}+2 \psi^{3}}\right)^{-1} \\
& \times \text { EllipticF }\left(\arcsin \sqrt{\frac{-\psi+r_{3}}{-r_{2}+r_{3}}}, \frac{r_{2}-r_{3}}{r_{1}-r_{3}}\right),
\end{aligned}
$$

where $Q$ is a constant of integration, $r_{1}, r_{2}$, and $r_{3}$ are the roots of the equation $1+2 P x^{2}+2 x^{3}=0$, and

$$
\begin{aligned}
r_{1}= & -\frac{P}{3}+\frac{2^{2 / 3} P^{2}}{3 \sqrt[3]{-27-4 P^{3}+3 \sqrt{81+24 P^{3}}}} \\
& +\frac{\sqrt[3]{-27-4 P^{3}+3 \sqrt{81+24 P^{3}}}}{3 \cdot 2^{2 / 3}} \\
r_{2} & =-\frac{P}{3}-\frac{(1+\sqrt{3} i) P^{2}}{3 \cdot 2^{1 / 3} \sqrt[3]{-27-4 P^{3}+3 \sqrt{81+24 P^{3}}}} \\
& +\frac{(1-\sqrt{3} i) \sqrt[3]{-27-4 P^{3}+3 \sqrt{81+24 P^{3}}}}{6 \cdot 2^{2 / 3}} \\
r_{3} & =-\frac{P}{3}-\frac{(1-\sqrt{3} i) P^{2}}{3 \cdot 2^{1 / 3} \sqrt[3]{-27-4 P^{3}+3 \sqrt{81+24 P^{3}}}}
\end{aligned}
$$

$$
+\frac{(1+\sqrt{3} i) \sqrt[3]{-27-4 P^{3}+3 \sqrt{81+24 P^{3}}}}{6 \cdot 2^{\frac{2}{3}}} .
$$

So, obviously, $2\left(\psi-r_{3}\right)\left(\psi-r_{1}\right)\left(\psi-r_{2}\right)=1+2 P \psi^{2}+2 \psi^{3}$. Then, (24) can be simplified as

$$
\xi+Q= \pm \sqrt{\frac{2 c}{r_{1}-r_{3}}} \text { Elliptic } F\left(\arcsin \sqrt{\frac{-\psi+r_{3}}{-r_{2}+r_{3}}}, \frac{r_{2}-r_{3}}{r_{1}-r_{3}}\right)
$$

where the Elliptic $F$ function, the elliptic integral of the first kind, is the inverse function of JacobiAmplitude function. Then, we get

$$
\begin{aligned}
\psi=\left(r_{2}-r_{3}\right) \sin ^{2}( & \text { JacobiAmplitude } \\
& \left.\times\left( \pm \sqrt{\frac{r_{1}-r_{3}}{2 c}}(\xi+Q), \frac{r_{2}-r_{3}}{r_{1}-r_{3}}\right)\right)+r_{3} .
\end{aligned}
$$

Replace $\psi$ with $e^{\phi}, \xi$ with $u+c v$ in (27), and then it is easy to obtain a family of explicit solutions for (1) as follows:

$$
\begin{aligned}
\phi=\ln \left[\left(r_{2}-r_{3}\right) \sin ^{2}(\right. & \text { JacobiAmplitude } \\
\times & \left( \pm \sqrt{\frac{r_{1}-r_{3}}{2 c}}\right. \\
& \left.\left.\left.\times(u+c v+Q), \frac{r_{2}-r_{3}}{r_{1}-r_{3}}\right)\right)+r_{3}\right],
\end{aligned}
$$

where $c$ and $Q$ are two arbitrary constants, and $r_{1}, r_{2}$, and $r_{3}$, as defined in (25), are the roots of the equation $1+2 P x^{2}+2 x^{3}=$ $0 ; P$ is also an arbitrary constant.

\section{Discussion and Comparison}

4.1. Solutions for Other Forms of Dodd-Bullough Equation. Other forms of Dodd-Bullough equation can also be dealt with using the same method. As examples, we propose the travelling-wave solutions for Dodd-Bullough-Mikhailov equation given by

$$
\phi_{u v}+e^{\phi}+e^{-2 \phi}=0
$$

and the Tzitzeica-Dodd-Bullough equation given by

$$
\phi_{u v}-e^{-\phi}-e^{-2 \phi}=0 .
$$


The two equations appear in problems varying from fluid flow to quantum field theory. For (29), the solution retrieved using our method is

$$
\begin{aligned}
\phi=\ln \left[\left(r_{2}-r_{3}\right) \sin ^{2}(\right. & \text { JacobiAmplitude } \\
\times & \left( \pm \sqrt{\frac{r_{3}-r_{1}}{2 c}}\right. \\
& \left.\left.\left.\times(u+c v+Q), \frac{r_{2}-r_{3}}{r_{1}-r_{3}}\right)\right)+r_{3}\right],
\end{aligned}
$$

where $c$ and $Q$ are two arbitrary constants and $r_{1}, r_{2}$, and $r_{3}$ are the roots of the equation $-1-2 P x^{2}+2 x^{3}=0 ; P$ is also an arbitrary constant.

And for (30), taking an assumption that $\phi^{\prime}=f\left(e^{-\phi}\right)$, and using a similar process, we can get a family of travelling-wave solution as follows:

$$
\begin{array}{r}
\phi=\ln \left(\frac { 1 } { 8 } \left((1+4 P) \frac{e^{\sqrt{2 P / c}(u+c v+Q)}}{P^{3 / 2}}\right.\right. \\
\left.\left.+\frac{e^{-\sqrt{2 P / c}(u+c v+Q)}}{\sqrt{P}}+\frac{2}{P}\right)\right),
\end{array}
$$

where $P, Q$, and $c$ are all arbitrary constants.

Actually, the method proposed in this lecture can be used to solve some more general forms of the Dodd-Bullough equation given by

$$
\phi_{u v}+\alpha e^{\phi}+\beta e^{-2 \phi}=0
$$

or

$$
\phi_{u u} \pm \phi_{v v}+\alpha e^{-\phi}+\beta e^{-2 \phi}=0 .
$$

It is easy to derive the solutions for these two general DoddBullough equations following the above listed steps. We would not go into the details.

4.2. Comparing of Previous Solutions. Compared to the previous works, we present new traveling-wave solutions for Dodd-Bullough.

Actually, in [14], Wazwaz proposes some travelling solutions for the Dodd-Bullough-Mikhailov and the TzitzeicaDodd-Bullough equations using the tanh method. It can be shown that the solutions for the Dodd-Bullough-Mikhailov given in [14] are only some special cases of our results.

For solutions of the Dodd-Bullough-Mikhailov equation given by (29), while we fix the constant $P$ to meet the parameter $\left(r_{2}-r_{3}\right) /\left(r_{1}-r_{3}\right)=1$, the JacobiAmplitude function will degenerate to an Arctan function. Then, we get

$$
\begin{aligned}
\phi=\ln [ & \left(r_{2}-r_{3}\right) \sin ^{2} \\
& \left.\times\left(-\frac{\pi}{2}+2 \arctan \left(e^{ \pm \sqrt{\left(r_{3}-r_{1}\right) / 2 c}(u+c v+Q)}\right)\right)+r_{3}\right] .
\end{aligned}
$$

That is,

$$
\phi=\ln \left[\left(r_{2}-r_{3}\right) \tanh ^{2}\left( \pm \sqrt{\frac{r_{3}-r_{1}}{2 c}}(u+c v+Q)\right)+r_{3}\right]
$$

Remembering that $r_{1}, r_{2}$, and $r_{3}$ are the roots of the equation $-1-2 P x^{2}+2 x^{3}=0$, and $\left(r_{2}-r_{3}\right) /\left(r_{1}-r_{3}\right)=1$, we can easily find that $r_{1}=r_{2}=-1$ and $r_{3}=1 / 2$; then, (36) changes into

$$
\phi=\left[\frac{1}{2}\left(1-3 \tanh ^{2}\left( \pm \frac{1}{2} \sqrt{\frac{3}{c}}(u+c v+Q)\right)\right)\right] .
$$

Then, it is easy to see that the solution of the Dodd-BulloughMikhailov equation given in [14] is a special case of ours.

Meanwhile, the solutions for Tzitzeica-Dodd-Bullough equation given by (30) are also different from previous results in $[7,13,14]$.

\section{Conclusion}

In this paper, we propose a new method to solve Dodd-Bullough equations under the assumption that $\phi^{\prime}=f\left(e^{\phi}\right)$. A new family of new explicit travelling-wave solutions for $\phi_{u v}=e^{\phi}-e^{-2 \phi}$ is derived. Using the proposed method, many other forms of Dodd-Bullough equations, Dodd-BulloughMikhailov, Tzitzeica-Dodd-Bullough, and some other general forms can be dealt with to get a new family of new explicit travelling-wave solutions. Compared with previous works, we can find that our solutions for Dodd-Bullough equations are different, and some previous results are special cases of ours.

\section{Acknowledgments}

This research is supported by National Natural Science Foundation of China (61171014, 61202436) and the Fundamental Research Funds for the Central Universities.

\section{References}

[1] R. K. Dodd and R. K. Bullough, "Polynomial conserved densities for the sine-Gordon equations," Proceedings of the Royal Society of London A, vol. 352, no. 1671, pp. 481-503, 1977.

[2] A. V. Žiber and A. B. Šabat, “The Klein-Gordon equation with nontrivial group," Doklady Akademii Nauk SSSR, vol. 247, no. 5, pp. 1103-1107, 1979.

[3] V. V. Nesterenko, "On the geometric origin of the equation $\phi_{11}-$ $\phi_{22}=e^{\phi}-e^{-2 \phi}$, Letters in Mathematical Physics, vol. 4, no. 6, pp. 451-456, 1980.

[4] V. V. Nesterenko, "Nonlinear $\sigma$ model for the Dodd-Bullough equation," Theoretical and Mathematical Physics, vol. 58, no. 2, pp. 126-131, 1984.

[5] A. V. Mikhailov, "The reduction problem and the inverse scattering method," Physica D, vol. 3, no. 1-2, pp. 73-117, 1981.

[6] A. R. Its, "Liouville's theorem and the inverse scattering method," Zapiski Nauchnykh Seminarov POMI, vol. 133, pp. 113125,1984 
[7] Y. V. Brezhnev, "Darboux transformation and some multi-phase solutions of the Dodd-Bullough-Tzitzeica equation: $U_{x t}=e^{U}$ $e^{-2 U}$, Physics Letters A, vol. 211, no. 2, pp. 94-100, 1996.

[8] I. Y. Cherdantsev and R. A. Sharipov, "Finite-gap solutions of the Bullough-Dodd-Zhiber-Shabat equation," Theoretical and Mathematical Physics, vol. 82, no. 1, pp. 155-160, 1990.

[9] I. Y. Cherdantzev and R. A. Sharipov, "Solitons on a finite-gap background in Bullough-Dodd-Jiber-Shabat model," International Journal of Modern Physics A, vol. 5, no. 15, pp. 3021-3027, 1990.

[10] A. J. Mohamad Jawad, M. D. Petković, and A. Biswas, "Modified simple equation method for nonlinear evolution equations," Applied Mathematics and Computation, vol. 217, no. 2, pp. 869877, 2010.

[11] E. M. E. Zayed, "A note on the modified simple equation method applied to Sharma-Tasso-Olver equation," Applied Mathematics and Computation, vol. 218, no. 7, pp. 3962-3964, 2011.

[12] E. M. E. Zayed and S. A. H. Ibrahim, "Exact solutions of nonlinear evolution equations in mathematical physics using the modified simple equation method," Chinese Physics Letters, vol. 29, no. 6, Article ID 060201, 2012.

[13] K. Khon and M. A. Akbar, "Exact and solitary wave solutions for the Tzitzeica-Dodd-Bullough and the modified KdV-ZakharovKuznetsov equations using the modified simple equation method," Ain Shams Engineering Journal, 2013.

[14] A. M. Wazwaz, "The tanh method: solitons and periodic solutions for the Dodd-Bullough-Mikhailov and the TzitzeicaDodd-Bullough equations," Chaos, Solitons and Fractals, vol. 25, no. 1, pp. 55-63, 2005.

[15] Y. C. Hon and E. Fan, "Soliton solutions and doubly periodic wave solutions for a new generalized Hirota-Satsuma coupled system," Applied Mathematics and Computation, vol. 146, no. 23, pp. 813-827, 2003. 


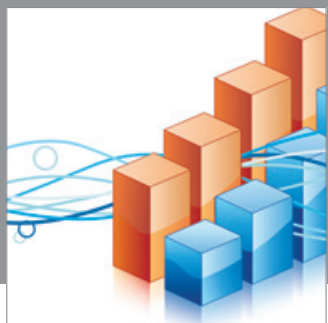

Advances in

Operations Research

mansans

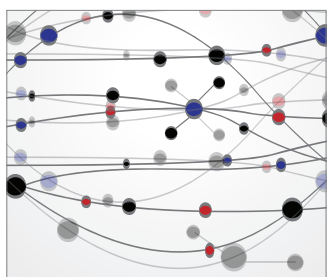

The Scientific World Journal
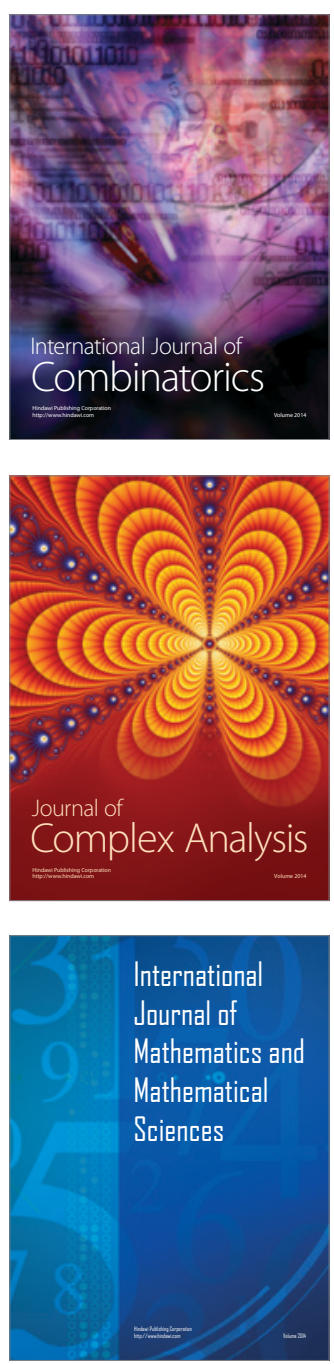
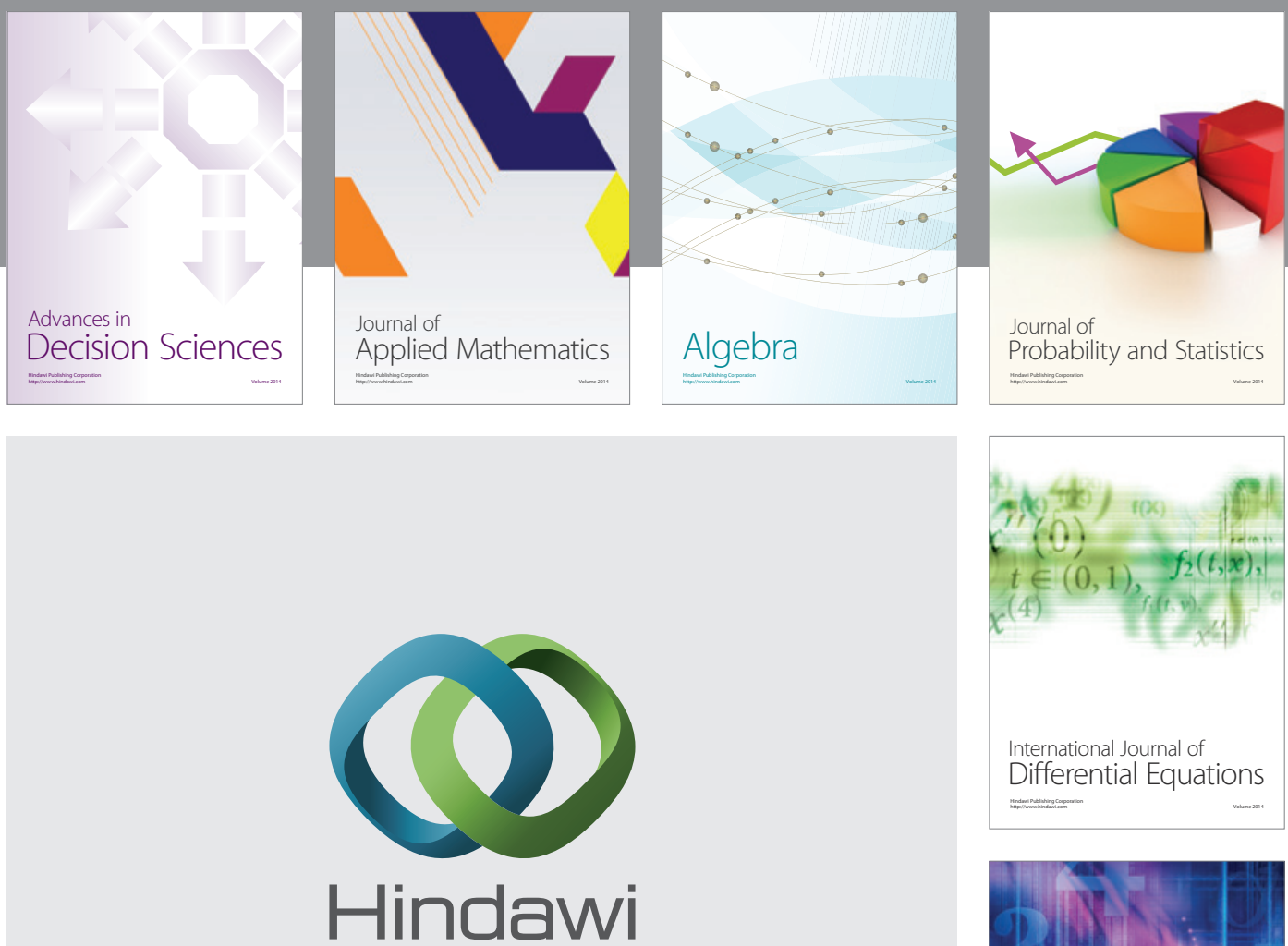

Submit your manuscripts at http://www.hindawi.com
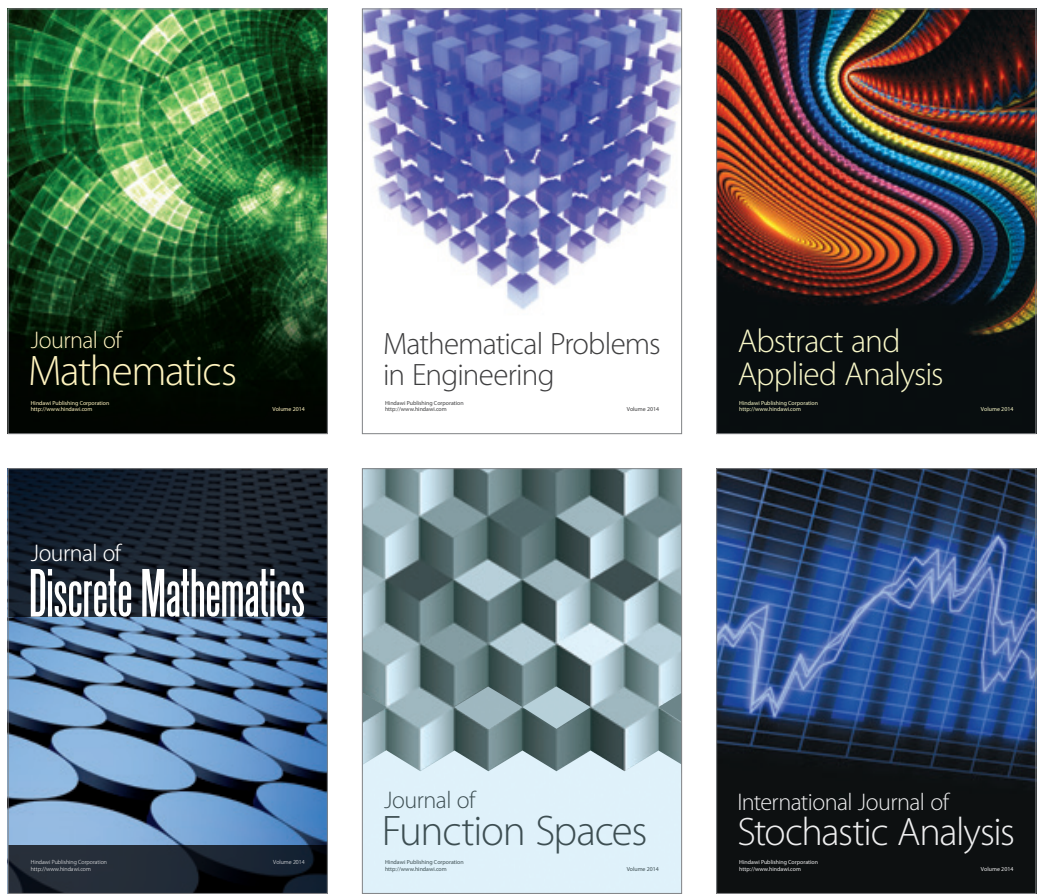

Journal of

Function Spaces

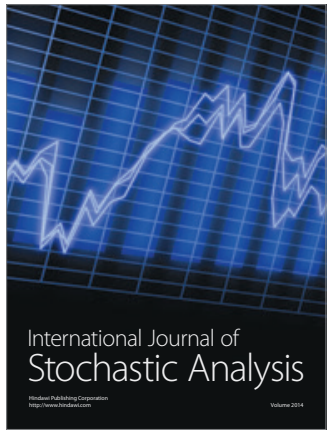

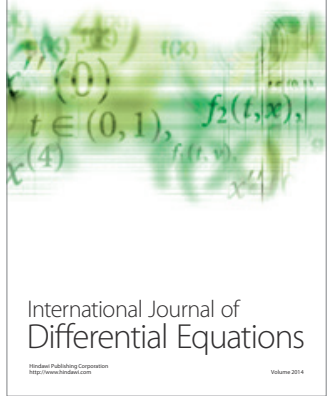
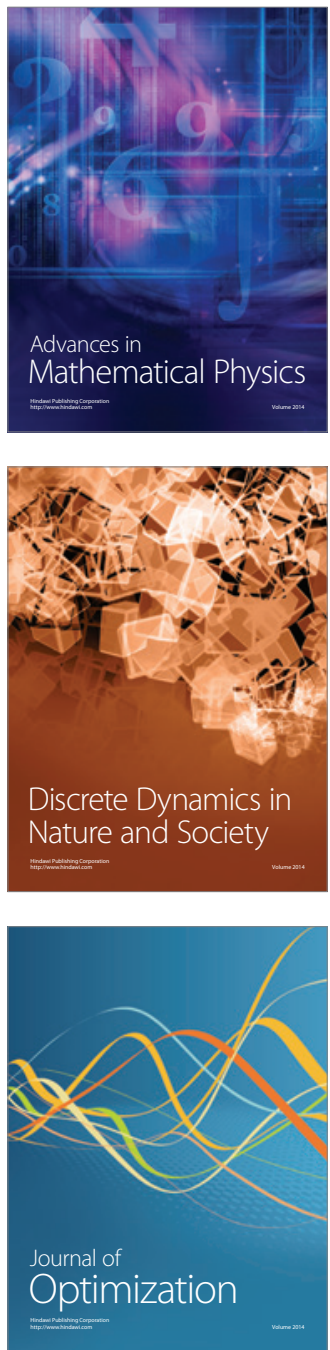